\title{
Estudo comparativo de excipientes em diferentes técnicas de preparação de comprimidos de cloridrato de propranolol ${ }^{1}$
}

\section{Comparative study of excipients for propanolol hydrochloryde tablets prepared by means of diferent techniques}

\author{
Marcela Maria Baracat ${ }^{2}$, Marlene Maria Fregonezi Nery², Edilaine de Freitas Gouveia ${ }^{3}$, Luciana \\ Meneguelli ${ }^{4}$, Silvia Michico Hizuka ${ }^{4}$, Cinara Maistro Mamprim ${ }^{51 a}$
}

Resumo: A hipertensão arterial sistêmica (HAS) é um dos mais importantes fatores de risco cardiovascular uma vez que contribui, mundialmente, com mais de 500 mil casos de acidentes cerebrovasculares (AVC), 150 mil mortes por hemorragia cerebral e aproximadamente um milhão de infartos de miocárdio (IAM). No Brasil estima-se que cerca de $15 \%$ dos indivíduos adultos possam ser rotulados como hipertensos. A hipertensão pode ser prevenida com a mudança no estilo de vida, embora na maioria dos casos se faça necessário o tratamento farmacológico. O cloridrato de propranolol é o fármaco de escolha para hipertensos idosos que tiveram infarto prévio do miocárdio. Os comprimidos podem ser preparados por três técnicas de fabricação diferentes. A técnica de granulação úmida é a mais comumente utilizada. Com o avanço de novos excipientes disponíveis no mercado para as Indústrias Farmacêuticas, tornou-se possível o emprego de técnica mais simples e econômica, melhorando a estabilidade física e química do produto, atingindo o objetivo de obter medicamentos eficazes e seguro. O objetivo deste trabalho foi desenvolver formulações de cloridrato de propranolol variando-se os excipientes e técnicas de fabricação. Os mesmos foram armazenados em estufa a 37 e $50^{\circ} \mathrm{C}$ com $90 \%$ UR durante 90 dias e analisados em intervalos de tempo preestabelecidos. As formulações foram avaliadas em função dos aspectos físicos e físico-químico.

Palavras-chave: cloridrato de propranolol, comprimido, granulação via úmida, compressão direta.

\begin{abstract}
Abstrast: Systemic arterial hipertension (SAH) is one of the major factors in cardiovascular risk, since it contributes to the existence of more than 500 thousand cases of cerebral vascular accidents (CVA), 150 thousand deaths by cerebral hemorrhage and approximately a million myocardium infarctions (IAM). In Brazil, it is estimated that about $15 \%$ of the adult population can be considered hypertensive. Hypertension can be prevented by changes in lifestyle, although in most cases, the treatment with drugs becomes necessary. Propranolol hydrochloride is the drug chosen for the hypertensive elderly population who has had myocardium infarctation previously. The drug is commercially available as injections, solutions, capsules and tablets. Tablets can be prepared using three different techniques. The most used technique is the granulation by moisture. With the advance of new excipients available in the market for the Pharmaceutical Industry, a more simple and economical technique became possible, improving the physical and chemical stability of the product, reaching the goal of getting more efficient and safer medicine. The purpose of this study was to develop formulations of propranolol hydrochloride tablets through the variation of excipients and manufacture techniques. The propranolol hydrochloryde tablets were stored at $37^{\circ} \mathrm{C}$ and $50^{\circ} \mathrm{C}$ with $90 \%$ UR for 90 days, and analysed in pre-established time intervals the formulations were evaluated as for the physical and physical-chemical aspects.
\end{abstract}

Key words: propranolol hydrochloride, tablet, wet granulation, direct compression.

\section{Introdução}

A hipertensão arterial sistêmica (HAS) é uma patologia clínica de natureza multifatorial caracterizada por níveis de pressão arterial (PA) elevados, sendo um dos mais importantes fatores de risco cardiovascular contribuindo, mundialmente, com mais de 500 mil acidentes cerebrovasculares (AVC), 150 mil mortes por hemorragia cerebral e aproximadamente um milhão de infartos do miocárdio (IAM). No Brasil, estima-se que cerca de $20 \%$ dos indivíduos adultos possam ser rotulados como hipertensos, aumentando essa ocorrência com a idade. A HAS multiplica o risco de danos cardiovasculares, o que contribui para aumentar a mortalidade e os custos sociais como invalidez e absenteísmo ao trabalho (BODANESE, 1998; EPIDEMIOLOGIA, 2001, p.12; FRANCISCHETTI; OIGMAN, 1987). O controle adequado dessa situação reduz significativamente os riscos individuais e os custos sociais (NASH, 1996).

O objetivo do tratamento da hipertensão arterial é reduzir a morbidade e mortalidade cardiovascular. $\mathrm{O}$ tratamento tem demonstrado ser particularmente útil para prevenir acidentes cerebrovasculares, preservar a função renal e prevenir ou retardar a progressão da insuficiência cardíaca.

\footnotetext{
1 Trabalho realizado no Laboratório de Produção de Medicamentos da Universidade Estadual de Londrina.

${ }^{2}$ Docente do Departamento de Tecnologia de Alimentos e Medicamentos da Universidade Estadual de Londrina.

${ }^{3}$ Farmacêutica do Laboratório de Produção de Medicamentos da Universidade Estadual de Londrina.

${ }^{4}$ Acadêmica do Curso de Farmácia e Bioquímica da Universidade Estadual de Londrina.

${ }^{5}$ Bolsista do CNPq, Departamento de Tecnologia de Alimentos e Medicamentos da Universidade Estadual de Londrina.
} 
A mudança do estilo de vida tem demonstrado ser efetiva em reduzir a pressão arterial, embora torne-se necessária a associação de tratamento farmacológico (FRANCISCHETTI; OIGMAN, 1987).

Os beta-bloqueadores como, por exemplo, o cloridrato de propranolol, são drogas de escolha para hipertensos idosos que tiveram infarto prévio do miocárdio, pois reduzem o risco de eventos subseqüentes e morte súbita (BODANESE, 1998). O fármaco apresenta-se comercialmente na forma de injetáveis e comprimidos.

O processo de fabricação de comprimidos em Indústria de Medicamentos dispõe de três técnicas de produção: granulação por via úmida (convencional), granulação por via seca e compressão direta (CD) (ANSEL et al, 1999; PRISTA et al, 1995).

O método de compressão direta oferece muitas vantagens como: simplicidade, economia, redução nas etapas de fabricação e aumento da estabilidade do produto (HSU et al., 1997; LIN, et al2001) quando comparado à técnica convencional, ou seja, granulação por via úmida.

A maioria dos fármacos não apresenta as propriedades de escoamento e alta compactabilidade necessárias para a compressão direta, havendo necessidade de adição de adjuvantes, os quais favorecem as ligações fracas entre as partículas, facilitando a coesão do material e conseqüentemente a compressão (MAARSCHALK et al., 1999). Porém, com o avanço de novos excipientes disponíveis no mercado para as Indústrias Farmacêuticas, tornou-se possível o emprego da técnica por CD. Os excipientes atualmente disponíveis contribuem, principalmente, facilitando a coesão entre as partículas, o escoamento do produto nas máquinas utilizadas e a desagregação dos comprimidos, sem a necessidade prévia de granulação, resultando em um menor tempo de produção e conseqüentemente diminuindo o custo final do produto (BARRA; SOMMA, 1996; CELIK, 1996; HSU et al, 1997; NASH, 1996; WILLIAMS et al., 1997).

Tradicionalmente, os excipientes estavam sendo definidos como substâncias inertes. No entanto, recentes trabalhos têm demonstrado que esta definição tornouse inadequada. Os excipientes farmacêuticos são substâncias adicionadas ao processo de fabricação para proteger e melhorar a biodisponibilidade, atribuindo segurança e eficácia ao produto durante o período de estocagem e uso (MORETON, 1996).

Devido ao alto índice de doenças cardiovasculares e conseqüentemente ao alto consumo de medicamentos, vários estudos têm sido direcionados para a fabricação de produtos com baixo custo para atender a população carente, porém com o compromisso de garantir a qualidade. Com essa finalidade, o trabalho propõe desenvolver formulações de comprimidos de cloridrato de propranolol, verificando o comportamento de diferentes excipientes pelas técnicas de granulação por via úmida e compressão direta.

\section{Material e Métodos}

\subsection{Matérias primas}

Todas as matérias-primas utilizadas foram de grau farmacêutico.

- Cloridrato de propranolol (Marty Chemicals B.L., Nertherlands)

- Lactose monohidratada (MEGGLE GMBH Meggiestr, Wasserburg)

- Manitol oral (Palmares-Ervy Comercial e Importadora Ltda., Brasil)

- Estearato de magnésio (Palmares-Ervy Comercial e Importadora Ltda., Brasil)

- Talco (Palmares-Ervy Comercial e Importadora Ltda., Brasil)

- Celulose microcristalina PH 102 (Mingtai Chemical Co., LTD., Taiwan)

- Celulose microcristalina PH 101 (Blanver Farmoquímica Ltda.,Brasil)

- Croscarmelose sódica (FMC Corporation, Philadelphia,PA)

- Amido Glicolato sódico (Palmares-Ervy Comercial e Importadora Ltda., Brasil)

- Amido de milho (RMB Refinações de Milho, Brasil Ltda)

\subsection{Reagentes}

Todos os reagentes utilizados nesta investigação foram grau analítico.

- Ácido clorídrico 1:100

- Metanol P.A.

- Solução karl fischer

- Padrão de cloridrato de propranolol

- Metanol secado

\subsection{Equipamentos}

- Misturador em V, capacidade $25 \mathrm{Kg}$ (FabbePrimar, SN 508)

- Máquina de compressão de 16 punções (Lawes, W-216)

- Balança (Toledo, 2124 - P10)

- Incubadora B.O.D. (Marconi , MA-414)

- Envelopadora (Fabrisul Duplex)

- Codificadora (Willet 3940

- Balança analítica (Mettler AE-200)

- Espectrofotômetro UV-VIS (Schmadzu 160-A)

- Titulador Karl Fischer (Mettler DL-35)

- Granulador oscilante (Lawes)

- Granulador rotativo (Lawes 919)

- Batedeira planetária (Amadio 150) 
- Estufa de secagem com circulação de ar forçada (Fabbe-Primar, LS 2000)

- Friabilômetro (Erweka TAR)

- Durômetro (Erweka TBH-30)

- Aparelho de dissolução (Erweka DT-6)

\subsection{Amostras}

Fórmulas 1, 2, 3, 4 e 5, apresentadas na Tabela 1 e preparadas conforme técnicas descritas a seguir.

- Técnicas de Preparação

As etapas utilizadas para o preparo do comprimido seguem os fluxogramas:

a) Granulação por via úmida

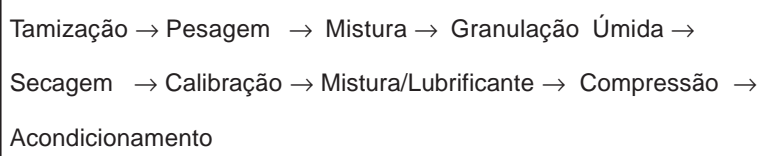

b) Compressão Direta

Tamização $\rightarrow$ Pesagem $\rightarrow$ Mistura/Lubrificante $\rightarrow$ Compressão Acondicionamento

- Análises

a) Métodos Físicos:

- Teste de Friabilidade

Pesaram-se 20 comprimidos, os quais foram submetidos a uma rotação de 25 rpm durante 4 minutos. Após este tempo calculou-se a resistência do comprimido a choque e atrito, conforme recomendação da Farmacopéia Americana (24. ed., 1999).

\section{- Teste de Dureza}

Obtido pela média aritmética da resistência $(\mathrm{Kp})$ de 10 comprimidos, determinado em durômetro a pressão contínua.

\section{- Teste de Dissolução}

O teste de dissolução foi realizado conforme recomendação da Farmacopéia Americana (24. ed.), 1999 utilizando o aparelho ERWEKA DT6 e os parâmetros: $1000 \mathrm{~mL}$ de ácido clorídrico (1:100), como meio de dissolução; aparato 1 (cesta), rotação de 100rpm; tempo de 30 minutos; tolerância (Q) de 75\% $+5 \%$; utilizando 6 comprimidos e temperatura de $37^{\circ} \mathrm{C}$ $\pm 0,5^{\circ} \mathrm{C}$. Após o tempo de agitação, coletou-se em torno de $25 \mathrm{ml}$ de solução de cada cuba. As soluções foram filtradas, a quantidade do princípio ativo foi determinada espectofotometricamente e calculada em relação ao padrão de cloridrato de propranolol preparado na mesma concentração. Os resultados obtidos foram analisados segundo os critérios de aceitação descritos abaixo: a- Estágio $\mathrm{E}_{1}$ : São testadas 6 unidades. Cada unidade individualmente deve apresentar resultado $\geq Q+5 \%$. Quando o estágio $E_{1}$ não for atendido seguir conforme item b,

b- Estágio $\mathrm{E}_{2}$ : São testadas mais 6 unidades. A média das 12 unidades testadas $\left(E_{1}+E_{2}\right)$ deve ser $\geq Q$ e nenhuma unidade deve apresentar resultados inferiores a $Q-15 \%$. Quando o estágio $E_{2}$ não for atendido seguir conforme item c,

c- Estágio $E_{3}$ : São testadas mais 12 unidades. A média das 24 unidades testadas $\left(E_{1}+E_{2}+E_{3}\right)$ deve ser $\geq Q$ e no máximo 2 unidades podem apresentar resultados inferiores a $Q-25 \%$.

\section{- Teste de Desintegração}

O teste foi realizado conforme recomendação da Farmacopéia Americana (24 ed., 1999) utilizando o aparelho ERWEKA ZT3 e água a $37^{\circ} \mathrm{C}$ como meio. $\mathrm{O}$ tempo de desintegração deve ocorrer no máximo em 30 minutos.

\section{b) Métodos Químicos:}

\section{Determinação do teor de princípio ativo}

O teor de princípio ativo nas amostras preparadas em solução na concentração teórica de $32 \mu \mathrm{g} / \mathrm{ml}$ de cloridrato de propranolol foi determinado por espectrofotometria no UV a $290 \mathrm{~nm}$ de acordo com a Farmacopéia Britânica (1988).

\section{c) Análise Estatística:}

O resultado de teor de cloridrato de propranolol foi submetido a análise estatística, através do cálculo de média, desvio padrão, análise de variância e teste de Tukey $(p<0,05)$ para verificar as diferenças entre as médias. Para processamento das análises utilizou-se o programa Statistical Analysis System (SAS) 1998.

\section{Resultados e Discussão}

As formulações em estudo, preparadas pela técnica de granulação por via úmida (fórmula 1 e 2) e por compressão direta (fórmula 3 a 5), descritas na Tabela 1, apresentaram características diversas em relação aos aspectos físicos de compressão.

A fórmula 1 contém em sua composição celulose microcristalina PH101, excipiente freqüentemente utilizado na fabricação de comprimidos, particularmente aconselhado por conferir um grau de aglutinação ao produto sem afetar as características de desagregação (WILLIAMS et al. 1997). Essas propriedades da celulose microcristalina influenciaram de maneira favorável nas características físicas dos comprimidos conforme resultados apresentados na Figura 1.

Analisando a referida Figura, observa-se que a fórmula 1 manteve as características de friabilidade e dureza durante 90 dias quando mantidos à temperatura de $37^{\circ} \mathrm{C} / 90 \%$ UR e $50^{\circ} \mathrm{C} / 90 \%$ UR. A fórmula 2 , que contém na formulação manitol, apresentou aumento 
significativo na porcentagem de friabilidade e diminuição dos valores de dureza que se acentuou com o aumento do tempo e temperatura de armazenamento (Figura 1).

As fórmulas 3 e 4 foram obtidas por compressão direta, e diferem na escolha do diluente (Tabela 1). A celulose microcristalina $\mathrm{PH} 102$, presente nas formulações, é utilizada na técnica citada, por suas partículas estarem na forma de granulado, fornecendo melhor fluidez para o produto, uniformidade de peso e menor força de compressão. A lactose presente na fórmula 3 é um excelente diluente que, além de suas propriedades redutoras, muito recomendáveis na maioria dos casos, apresenta propriedade de compatibilidade e menor poder aglutinante. Os resultados demonstraram que a friabilidade e dureza mantiveram-se compatíveis com as exigências. A fórmula 4, na qual se utilizou manitol em substituição a lactose, comportou-se de maneira diferente. Durante o processo produtivo os pós não foram capazes de fluir através do funil para o interior da matriz, impossibilitando a compactação do produto.

As fórmulas 3 e 5 diferem na escolha do desagregante. Na fórmula 3 utilizou-se croscarmelose sódica que é uma carboximetilcelulose sódica modificada e apresenta um alto poder desagregante até mesmo em baixos níveis, podendo ser utilizada na concentração de 0,5 a 3\%, inclusive em comprimidos e grânulos de elevada dureza. A presença de croscarmelose sódica tem demonstrado excelente estabilidade de prateleira em grande número de formulações. A taxa de absorção de água e o grau de entumescimento representam importantes perfis para determinar desempenho dos desagregantes. Esta dupla funcionalidade traduz um alto poder de desagregação em baixos níveis de concentração, quando comparada com outros desagregantes (FERRERO etal, 1997; AC-DI-SOL, 1995). Entretanto, com o glicolato amido sódico, é necessário 4 a $8 \%$ para oferecer propriedade de desagregação. De acordo com Chang et al, 1998, altas concentrações de desagregante favorecem a absorção de água do ambiente, influenciando negativamente nas características de friabilidade e dureza conforme Figura 1. Os resultados dos testes de dissolução, de desintegração e de teor não apresentaram alterações nas condições estudadas, conforme descritos na Tabela 2 e 3.

\section{Conclusão}

Pelo estudo comparativo das formulações pela técnica de granulação a úmido e por compressão direta, foram selecionadas as fórmulas 1 e 3, respectivamente, por manterem a estabilidade desde o início do estudo. Comparando os resultados, a fórmula 3 apresenta a vantagem de tornar o processo simples, pois elimina a fase de granulação, operação que implica outras etapas como secagem e calibração.

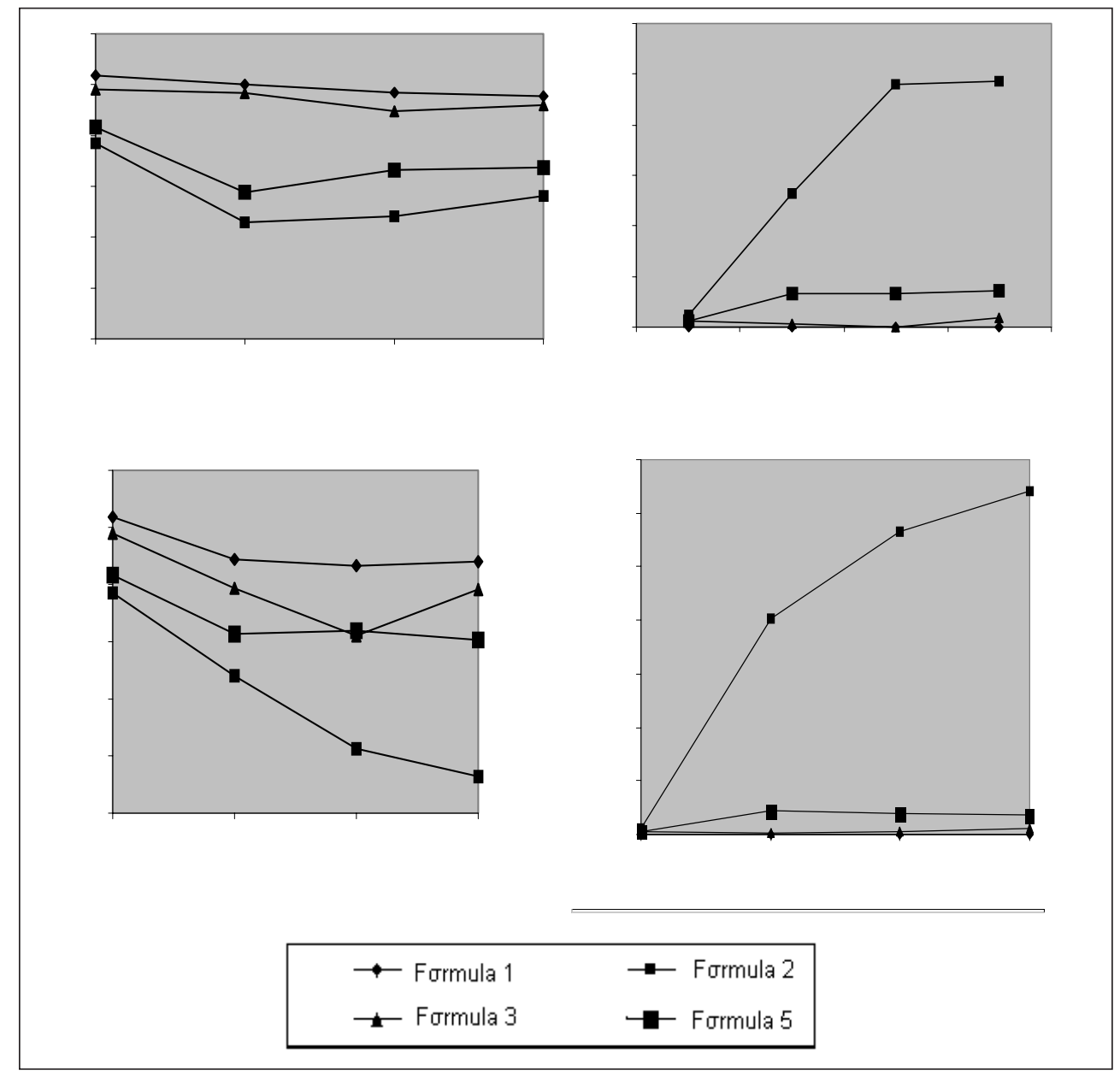

Figura 1 - Valores de dureza e friabilidade das fórmulas 1, 2, 3 e 5, armazenadas a temperatura de $37^{\circ} \mathrm{C} \mathrm{e}$ $50^{\circ} \mathrm{C}$ com $90 \%$ UR, durante 90 dias. 


\section{Referências}

AC-DI-SOL Croscarmellose Sodium. FMC Corporation. 1995. [Catálogo].

ANSEL, H. C.; POPOVICH, N. G.; ALLEN Jr., L. V. Farmacotécnica - formas farmacêuticas e sistema de liberação de fármacos. 6. ed. [S. I.]: Editorial Premier, 1999. p.201-227.

BARRA, J., SOMMA, R. Influence of the physicochemical variability of magnesium stearate on its lubricant properties: possible solutions. Drug Dev. Ind. Pharm., New York, v.22, n.11, p.1105-1120, 1996.

BODANESE, L.C. Hipertensão arterial no idoso. Rev. Bras. Clin. Terap. Porto Alegre, v.24, n.5, p.194-199, 1998.

CELIK, M. The past, present, and future of tableting technology. Drug Dev. Ind. Pharm., New York, v.22, n. 1, p.1-10, 1996.

CHANG, R-K et al. Evaluation of the disintegrant properties for na experimental, crosslinked polyalkylammonium polymer. Int. J. Pharm., v.173, p.87-92, 1998.

EPIDEMIOLOGIA. Cadernos de Atenção Básica, Brasília, n.7, p.11-13, 2001.

FERRERO, $\mathrm{C}$ et al. Disintegrating efficiency of croscarmellose sodium in a direct compression formulation. Int. J. Pharm., v.147, p.11-21, 1997.
FRANCISCHETTI, E. A., OIGMAN, W. Hipertensão arterial. Rev. Bras. Med., Rio de Janeiro, v.44, n.10, p.51-74, 1987.

HSU, S. H et al. Evatuation of era-tab as a direct compression excipient. Dru Dev. Ind. Pharm., New York, v.23, n.7, p.711-716, 1997.

LIN, K-H.; LIN S-Y.; LI M-J. Compression forces and amount of outer coating layer affecting the time-controlled desintegration of the compression-coated tablets prepared by direct compression with micronized ethylcellulose. $J$. pharm. Sci., v 90, n.12, p.2005-2009, 2001.

MAARSCHALK, K. VAN DER V., BOLHUIS, G. K. Aprimoramento das propriedades de materiais para compressão direta. Pharm. Technol., v.3, n.4-8, p.17-25, 1999.

MORETON, R. C. Tablet excipients to the year 2001: a look into the crystal ball. Drug Dev. Ind. Pharm., New York, v.22, n.1, p.11-23, 1996.

$\mathrm{NASH}, \mathrm{R}$. A. Process validation: a 17-year retrospective of solid-dosage forms. Drug Dev. Ind. Pharm., New York, v.22, n.1, p.25-34, 1996.

PRISTA, L. N.; ALVES, A. C.; MORGADO, R. M. R. Técnica farmacêutica e farmácia galênica. 5. ed. Lisboa: Fundação Calouste Gulbenkian, 1995. v.1, p.325-478.

WILLIAMS, R. O.; SRIWONGJANYA, M.; BARRON, M. $\mathrm{K}$. Compaction properties of microcrystalline cellulose using tableting indices. Drug Dev. Ind. Pharm., New York, v.23, n.7, p.695-704, 1997.

Tabela 1 - Formulações de cloridrato de propranolol 40mg, desenvolvidas pelas técnicas de granulação por via úmida e por compressão direta. 
Tabela 2 - Valores referentes aos testes de desintegração, dissolução e teor apresentados pelas formulações de Cloridrato de Propranolol $40 \mathrm{mg}$, fórmulas 1, 2, 3 e 5, armazenadas em temperatura de $37^{\circ} \mathrm{C}$ e umidade relativa de $90 \%$, durante 90 dias.

\begin{tabular}{cccc} 
\pm & \pm & \pm & \pm \\
\pm & \pm & \pm & \pm \\
\pm & \pm & \pm & \pm \\
\pm & \pm & \pm & \pm \\
\pm & \pm & \pm & \pm \\
\pm & \pm & \pm & \pm \\
\pm & \pm & \pm & \pm \\
\pm & \pm & \pm & \pm \\
\hline
\end{tabular}

*Médias do tempo de desintegração, duas repetições;

${ }^{* *}$ Médias da \% dissolvida de cloridrato de propranolol das 6 unidades realizadas no estágio 1 (E1), onde Q =75 + 5\%; ${ }^{* * *}$ Médias dos teores de cloridrato de propranolol em triplicata de cada formulação com desvio padrão entre parênteses. Médias com letras iguais na mesma coluna não diferem significativamente pelo teste de Tukey ao nível de $5 \%$.

Tabela 3 - Valores referentes aos testes de desintegração, dissolução e teor apresentados pelas formulações de Cloridrato de Propranolol $40 \mathrm{mg}$, fórmulas 1, 2, 3 e 5, armazenadas em temperatura de 50ํ e umidade relativa de $90 \%$, durante 90 dias.

*Médias do tempo de desintegração, duas repetições;

**Médias da \% dissolvida de cloridrato de propranolol das 6 unidades realizadas no estágio 1 (E1), onde Q =75 + 5\%;

***Médias dos teores de cloridrato de propranolol em triplicata de cada formulação com desvio padrão entre parênteses. Médias com letras iguais na mesma coluna não diferem significativamente pelo teste de Tukey ao nível de $5 \%$. 